\title{
Selected topics from the 24th international colloquium on the dynamics of explosions and reactive systems, Taipei, Taiwan, July 28-August 2, 2013
}

\author{
G. Ciccarelli ${ }^{1}$
}

Published online: 31 March 2015

(C) Springer-Verlag Berlin Heidelberg 2015

This issue contains selected papers that were presented at the 24th international colloquium on the dynamics of explosions and reactive systems (ICDERS) held in Taipei, Taiwan July 28-August 2, 2013. The biennial ICDERS is the reference meeting focusing specifically on dynamic aspects of reactive systems. The local organization was headed by Steven S. Shy (National Central University, Taiwan) and Yei-Chin Chao (National Cheng-Kung University, Taiwan). The program committee was chaired by A. Koichi Hayashi (Aoyama Gakuin University, Japan) and co-chaired by Francesco S. Marra (CNR Istituto di Ricerche sulla Combustione and Universita del Sannio, Italy) and Eric L. Petersen (Texas A\&M University, USA), with the assistance of Jenny Chao (FM Global, USA), Nabiha Chaumeix (CNRS Orleans, France), Robert K. Cheng (Lawrence Berkeley National Laboratory, USA), Gaby Ciccarelli (Queen's University, Canada), Gaetano Continillo (Universita del Sannio Benevento, Italy), Derek DunnRankin (Department of Mechanical Engineering, University of California, Irvine), Andrew Higgins (McGill University, Canada), Scott I. Jackson (Los Alamos, USA), Mike Kuznetsov (Karlsruhe Institute of Technology, Germany), Ulrich Maas (Karlsruhe Institute of Technology, Germany), Akiko Matsuo (Keio University, Japan), Makihito Nishioka (University of Tsukuba, Japan), Elaine S. Oran (University of Maryland, USA), Oleg G. Penyazkov (Belarus Academy of Sciences, Belarus), Steven S. Shy (National Central University, Taiwan), Nobuyuki Tsuboi (Kyushu Institute

\footnotetext{
G. Ciccarelli

ciccarel@me.queensu.ca

1 Department of Mechanical and Materials Engineering, Queen's University, 130 Stuart Street, McLaughlin Hall, Kingston, ON K7L 3N6, Canada
}

of Technology, Japan), Jianping Wang (Peking University, China), Jennifer X. Wen (Kingston University, UK), Vigor Yang (Georgia Tech, USA) and Fan Zhang (DRDC Suffield, Canada).

Roughly 300 participants attended the conference and delivered 239 oral presentations and presented over 50 posters on explosions, detonations, ignition, turbulent combustion and other topics in combustion. With 27 countries represented, the conference is truly international. As in previous years, presenters at the conference were encouraged to submit their work to a selection of three journals depending on the research topic. The current issue includes papers dealing with combustion in high-speed flow and shock waves, detonation and deflagration-to-detonation transition, whose authors elected to submit to Shock Waves. Submissions were subjected to the same very rigorous review process as a regular submission to Shock Waves. Each paper was reviewed by at least two independent referees, each of which is an internationally recognized expert in the field. Twelve papers from the conference were submitted, of which three were rejected and two were withdrawn by the authors after an initial "major revision" decision. The remaining seven papers were eventually accepted, upon two or more revisions, after a consensus was reached by the referees and the editor. The current issue includes papers on ignition delay time behind a reflected shock, DME detonation cell structure, slipstream stability for a triple-shock configuration, rapid boiling of carbon dioxide, and three papers on combustion in heterogeneous media (involving liquid spray or inert particles). The last paper in the issue dealing with Mach reflection of a detonation wave did not originate from the conference but is included due to its high relevance to the topics covered at ICDERS. Geographically, papers from the conference originated from the USA (one), Norway (one), Canada (two), and Russia (three). 
I wish to thank the authors who submitted their papers to this issue and then revised their papers numerous times in order to produce the best quality possible. I would also like to thank the many reviewers whose help was crucial in ensuring the high quality of the papers that appear in this issue. 